\title{
Telemedizin weltweit mit einem offenen und verteilten Telemedizinsystem
}

\author{
K. Brauchli , L. Banach ${ }^{b}$, P. Dalquen ${ }^{a}$, G. Haroskec ${ }^{c}$, N. Hurwitz ${ }^{a}$, K.-D. Kunzed, G. Jundt ${ }^{a}$, H. Oberlie, G. Stauch ${ }^{f}$, P. Went ${ }^{a}$, M. Oberholzer ${ }^{a}$
}

\section{Einleitung}

Die Telepathologie startete in der Schweiz im Jahre 1991 [1]. Anlass dazu war die Frage: «Ist es möglich, mit den sich abzeichnenden neuen Kommunikationstechnologien einen intraoperativen Schnellschnittdienst des Instituts für Pathologie Basel für das Kreisspital Oberengadin, Samedan, einzurichten?» Verallgemeinert ging es um die Frage, ob es möglich ist, in Zukunft vermehrt Wissen und Erfahrung von Zentren in periphere Institutionen zu transferieren.

Neun Jahre später (im Sommer 2000) fragten die Chirurgen des National Referral Hospital, Solomon Islands (Südpazifik), an, ob es inzwischen nicht doch möglich geworden sei, auch in Honiara in schwierigen Fällen mit Hilfe von Telemedizin schneller als bis anhin zu einer pathologisch-anatomischen Diagnose zu kommen.

Die während der letzten drei Jahre verstärkten Anstrengungen im Forschungsprojekt «Telemedizin» am Institut für Pathologie der Universität Basel richteten sich in erster Linie auf die Entwicklung von Grundlagen für eine einfach zu praktizierende, vielfältig einsetzbare, plattformunabhängige und kostengünstige Telemedizin. Parallel dazu wurden die gewonnenen Erkenntnisse und Modelle in ein konkretes Telemedizinsystem umgesetzt.

a Institut für Pathologie der Universität Basel, Basel, Schweiz.

b Institut für Pathologie der Universität Transkei, Umtata, Südafrika.

c Krankenhaus Dresden-Friedrichstadt, Dresden, Bundesrepublik Deutschland.

d Institut für Pathologie der Technischen Universität, Dresden, Bundesrepublik Deutschland.

e National Referral Hospital, Honiara, Solomon Islands.

f Institut für Pathologie, Aurich, Bundesrepublik Deutschland.

Korrespondenz:

Prof. Dr. med. Martin Oberholzer Kantonsspital

Institut für Pathologie

Schönbeinstrasse 40

CH-4003 Basel geht, sind Informationen, die in Form von Bildern oder Kurzfilmen (z.B. Ultraschallsequenzen, Echokardiographie) darstellbar sind. Die Bilder müssen bereitgestellt, übertragen und archiviert werden. Weil in allen Fachdisziplinen der Medizin Bildinhalte diagnostisch relevant sein können, ist eine Unterscheidung von Spezialdisziplinen in der Telemedizin eigentlich nicht nötig, ausser wenn man die Bedingungen, unter welchen die Bilder generiert werden, speziell erwähnen will (z. B. Teleradiologie, Teledermatologie, Telepathologie). Videoconferencing in der Medizin kann als «Telemedizin im weitesten Sinne» verstanden werden, ebenso die medizini- sche Fernüberwachung. Diese beiden Teilgebiete wurden aber in der vorliegenden Studie nicht behandelt.

Das Spezifische an der Telemedizin besteht im Gegensatz zu anderen Formen der Kollaboration in der Medizin darin, dass verschiedene Partner einen Dienst an einem Patienten leisten, ohne dass sie alle am Ort, wo der Patient sich befindet, anwesend sein müssen.

Verschiedene Pilotversuche liessen erkennen, dass für eine erfolgreiche telemedizinische Kollaboration den Partnern folgende Infrastruktur zur Verfügung stehen muss:

1. Die Telemedizin muss vom gewohnten Arbeitsplatz aus, zu jeder Zeit, als Routineverfahren und spontan eingesetzt werden können.

2. Das System muss die Konsultation jedes beliebigen Partners über einen einfachen $\mathrm{Zu}$ gang zum benutzten Netzwerk ermöglichen.

3. Das System muss einfach bedienbar sein.

Auf dem Hintergrund dieser Postulate lässt sich Telemedizin auf ein einfaches Modell mit drei Standbeinen reduzieren:

1. Einer der Partner ist der Nichtexperte, welcher einem oder mehreren Partnern eine oder mehrere Fragen unterbreitet.

2. Die kontaktierten Partner sind Experten, welche die Fragen beantworten.

3. Die Partner deponieren die Fragen und Antworten zusammen mit den Begleitinformationen (statische und dynamische Bilder, Textfiles, Toninformationen) auf einem Server und können dort die «Dokumente» einsehen.
Das Modell funktioniert, wenn drei Voraussetzungen erfüllt sind:

1. Die auf dem Server deponierten Informationen müssen von einer strukturierten Datenbank verwaltet werden.

2. Der Server muss über alle Aktionen der Partner (z.B. die aktuelle Position eines fernsteuerbaren Mikroskops) orientiert werden.

3. Den Partnern müssen zur Herstellung der Bilder Hilfsgeräte (Manipulatoren) zur Verfügung stehen, welche sie mit ihrem Computer leicht verbinden können. 
Abbildung 1

Modulare Struktur der Software von iPath (siehe Text).

Mit iPath kann auch Telemikroskopie betrieben werden. Dazu muss auf dem Computer des Nichtexperten (mit dem Mikroskop) das Modul Microscope-Controller installiert werden. Dieses Modul ist als freie Software verfügbar, kann auf jedem PC installiert werden und passt zu verschiedenen Typen von Mikroskopen.

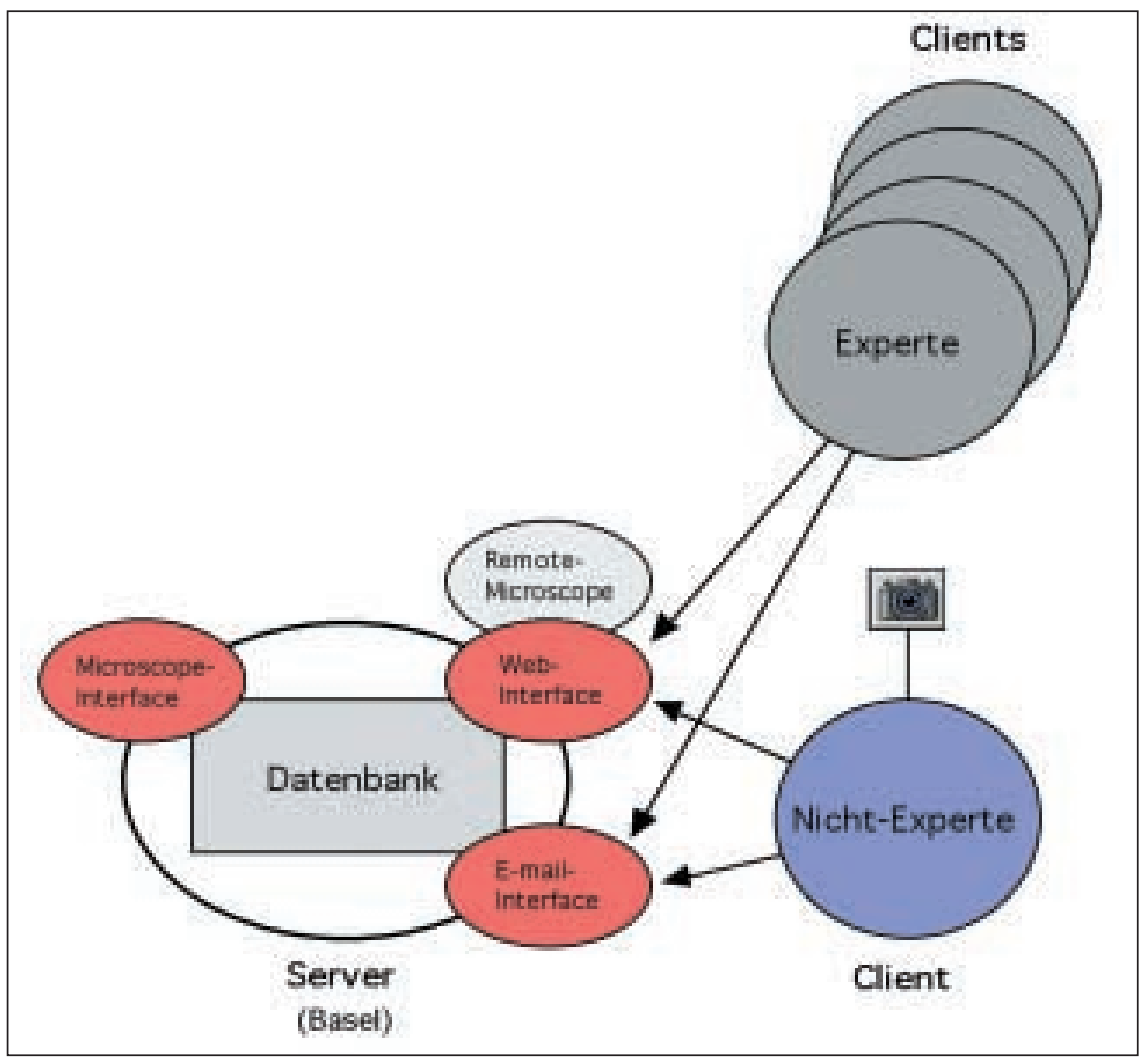

Im System iPath (http://telepath.patho.unibas.ch) ist das aufgezeigte Modell realisiert und sind die erwähnten Bedingungen berücksichtigt. iPath steht als Open-source-Software zur Verfügung, kann auf beliebigen Servern installiert werden und ist modular aufgebaut (Abb. 1). Die Hardwaremodule sind: ein zentraler Server im Internet [2] mit der Datenbank und den Softwaremodulen (siehe unten), ein Computer (PC oder MacIntosh) und eine Bildquelle (Digitalkamera oder Videokamera kombiniert mit einem Bildwandler [Frame Grabber], Ultraschallgerät, Röntgengerät, Mikroskop verbunden mit einer Kamera). Der Bildwandler wandelt das gewöhnliche Videobild in ein digitales Bild um. Mit iPath ist jeder Bildwandler mit dem Standard «Video for Windows» (VfW) kompatibel. Die Softwaremodule sind: das Web-Interface, welches auch das Modul Remote-Microscope für die Telemikroskopie enthält, das E-mail-Interface und das Microscope-Interface (Abb. 1). Für die Telemikroskopie mit iPath benötigt der Nichtexperte noch ein weiteres Modul (Microscope-Installer) auf seinem Computer.
Die Datenbank, welche iPath zugrunde liegt, besteht aus Gruppen, z.B. «Cardiology Basel», «Pathologie Jura», «South Pacific», «Bangladesh»). Diese Gruppen enthalten die Fälle, welche

1. von den Nichtexperten den Experten vorgestellt werden oder

2. an einem Kolloquium (z.B. Onkologiebesprechung) diskutiert werden sollen.

Ein Fall entspricht formal einer elektronischen Krankengeschichte mit Texten, Bildern und Kurzfilmen. Jeder Fall setzt sich zusammen aus den Feldern zur Identifikation, dem Feld «Beschreibung» (freier Text), der Bildgalerie, welche beliebige Dokumente (.jpg, .txt, .xls, fp3, .pdf, .mp3), Formulare (.html), Gruppen von Objekten (z.B. statische Bilder) und Kurzfilme enthalten kann, und dem Feld «Kommentare» (freier Text) (Abb. 2). $\mathrm{Zu}$ jedem Fall können von den Mitgliedern der Gruppe, in der sich der Fall befindet, beliebig viele Kommentare geschrieben werden.

Der Zugang der Benützer zu iPath erfolgt in vier Schritten:

1. Im ersten Schritt wird die Internetadresse http://telepath.patho.unibas.ch) aufgerufen;

2. im zweiten Schritt registriert sich der interessierte Benutzer auf der Homepage von iPath;

3. dann folgt die computerunterstützte Aktivierung des Benützers in iPath;

4. im vierten Schritt verschafft der Gruppenadministrator dem Benützer Zugang zu jenen Gruppen, in denen der Benützer gerne mitarbeiten möchte.

Von der Homepage aus können die einzelnen Gruppen und ihre Aktivitäten eingesehen werden. Nach der Anmeldung in iPath (über Name und Passwort) wird dem Benützer als erstes eine Tabelle mit den Gruppen präsentiert, zu denen er Zutritt hat. Mit der Registrierung erhält jeder Benützer automatisch Zugang zur Gruppe «Test».

Jeder Benützer kann in jeder Gruppe, zu der er Zugang hat, einen Fall neu eröffnen und dem Fall die entsprechenden Informationen als freien Text (in den dafür vorgesehenen Feldern) oder über die Funktion «add» (siehe Abb. 4) in Form von Bildern, Kurzfilmen oder anderen Dokumenten beigeben. Der Fall kann nur von derjenigen Person, welche ihn eröffnet hat, verändert werden, ausgenommen, die Person erlaubt als «Eignerin» des Falles anderen Mitgliedern der Gruppe, dem Fall weitere Dokumente zuzufügen. Dies ist dann wichtig, wenn z. B. bei einer Onkologiebesprechung gleichzeitig Bilder aus der Radiologie, Zytologie und Histologie zur Ver- 
Abbildung 2

Struktur eines Falles.

Der Fall entspricht einer elektronischen Krankengeschichte. Diese besteht aus vier Teilen: der Fallidentifikation, einer Beschreibung (Description), einer Bildgalerie (Images) und den Kommentaren (Comments). Dargestellt ist ein Beispiel aus einer Onkologiebesprechung am Kreiskrankenhaus Lörrach (Bundesrepublik Deutschland). Die Histologiebilder wurden vom Institut für Pathologie der Universität Basel, die Röntgenbilder von der Abteilung für Radiologie des Kreiskrankenhauses beigesteuert. Unter «Comments» ist das Beschlussprotokoll festgehalten. Im Formular BASEL Report sind die Schlüsseldaten (Alter und Geschlecht des Patienten, Krankengeschichte, klinische Diagnose und pathologisch-anatomische Diagnose) aufgeführt.

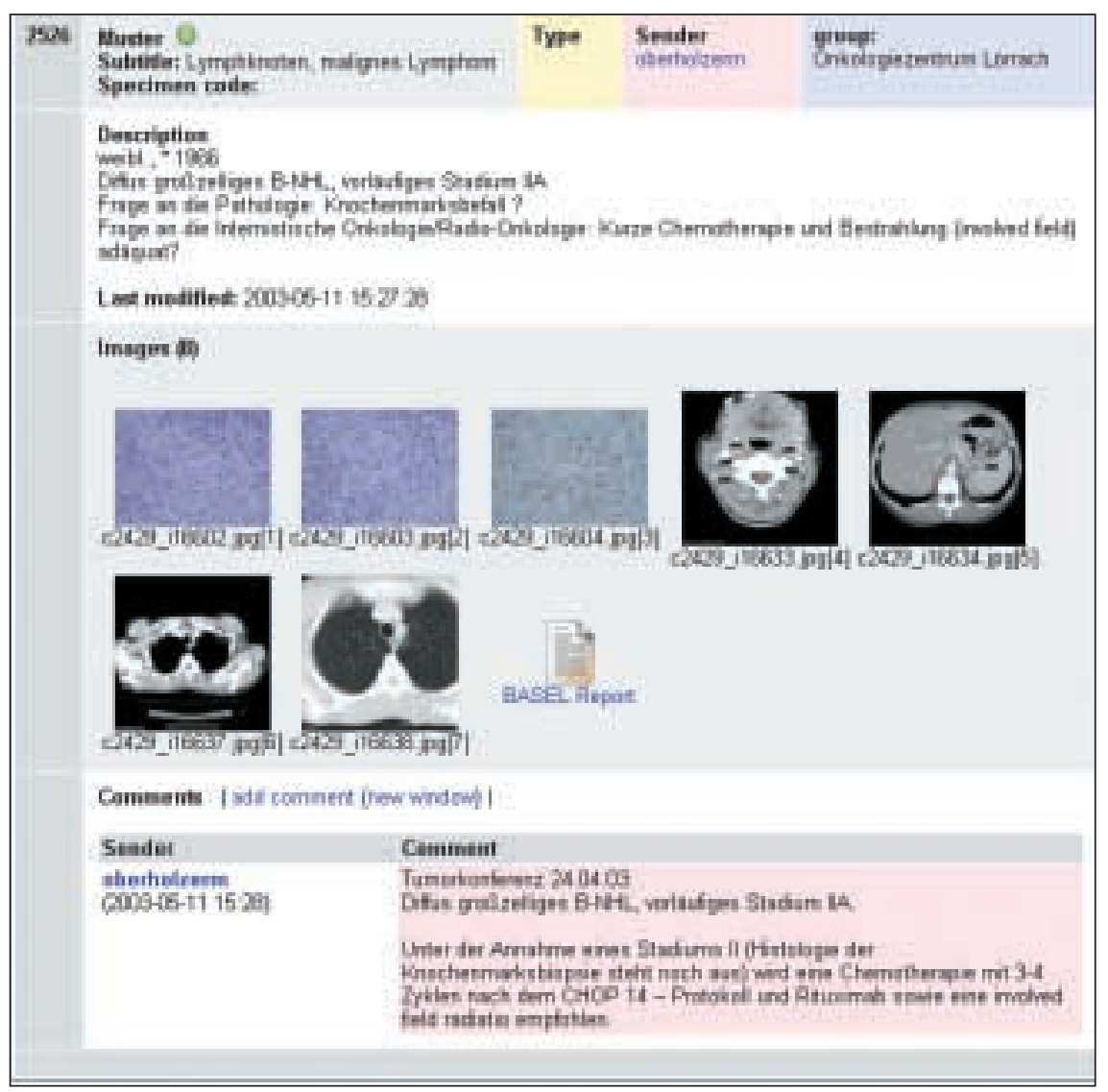

fügung stehen sollten. Jedes Mitglied der Gruppe kann aber unter «Comments» (Abb. 2) beliebig Kommentare zum Fall eingeben.

Die Eingabe von Text und Bildern in einen neuen Fall kann direkt über das Web-Interface oder indirekt über das E-mail-Interface erfolgen (siehe Abb. 1). Wenn das E-mail-Interface benützt wird, müssen vier Bedingungen berücksichtigt werden:

1. Der Absender muss registrierter iPath-Benützer sein.

2. Der Absender muss in iPath unter seinen «Preferences» festgelegt haben, in welcher Gruppe er die von ihm an iPath gesandten e-mails deponiert haben will.

3. Die vom Absender anlässlich seiner Registrierung in iPath deponierte e-mail-Adresse muss gleich lauten wie die e-mail-Adresse des Absenders.
4. Das E-Mail muss eine standardisierte Struktur aufweisen: Die Adresse ist «iPath@unibas.ch»; als «Bezeichnung» muss ein frei wählbarer Code eingegeben werden, welcher den Fall identifizieren lässt; als «Beschreibung» kann beliebig freier Text übermittelt werden; die Bilder werden als «angefügte Dokumente» gesandt.

Um das Verfahren gegen Missbrauch zu schützen, wird zusätzlich ein spezieller Code verlangt, welcher an einer vordefinierten Stelle einzugeben ist und erst nach eindeutiger Identifizierung des Benützers vom Gruppenadministrator mündlich dem Benützer mitgeteilt wird. Über 90\% der aus Entwicklungsländern und «Rural Countries» in iPath deponierten Konsiliarfälle werden via E-Mail an iPath gesandt.

\section{Material}

Nach einer ausgiebigen Testphase im Labor wurde iPath vor 2 Jahren im Internet freigegeben mit dem Ziel, zu beobachten, wie das neue Telemedizinkonzept grundsätzlich akzeptiert und für welche Anwendungen es eingesetzt wird. Inzwischen sind auf dem Server am Rechenzentrum der Universität Basel mehr als 60 Gruppen installiert worden, mehr als 500 Benützer haben sich registriert und mehr als 1700 Fälle wurden bearbeitet. Neben dem Server in Basel sind weitere Server in Deutschland, Norwegen, den USA und Südafrika in Betrieb. Die verschiedenen Anwendungsgebiete, welche sich im Lauf der Zeit ergeben haben, sind unter «Resultate» zusammengestellt.

Neben den möglichen Anwendungen, für die iPath eingesetzt werden kann, liess sich in den ersten 2 Jahren des Einsatzes von iPath auch überprüfen, welche Ausrüstung für eine erfolgreiche Arbeit mit iPath minimal erforderlich ist. Ein Nichtexperte (welcher Fragen stellt) braucht einen Computer (PC oder MacIntosh), einen Internetanschluss mit einem Web-Browser (Explorer 5 oder Netscape 6) und eine Bildquelle (z.B. Digitalkamera oder Videokamera), welche mit dem Computer verbunden werden kann. Die Experten (welche Fragen beantworten) benötigen ausschliesslich einen Computer mit einem Internetanschluss und einem Web-Browser (siehe oben). 
Abbildung 3

Einsatzgebiete von iPath.

iPath wurde nur zur Hälfte der Fälle (dargestellt mit den ersten drei Säulen) für pathologisch-anatomische Diagnosen eingesetzt. Unter der Rubrik «Andere» befinden sich auch Schnittseminare und die Fälle, welche von der deutschsprachigen Arbeitsgruppe für Knochentumoren bearbeitet und diskutiert werden. Die Gruppe «erste Meinung» umfasst jene Fälle, welche von Ärztinnen und Ärzten aus Spitälern eingereicht wurden, welche über keinen Pathologen vor Ort, sondern nur über ein einfaches Histologielabor verfügen (z. B. Honiara, Solomon Islands, Südpazifik).

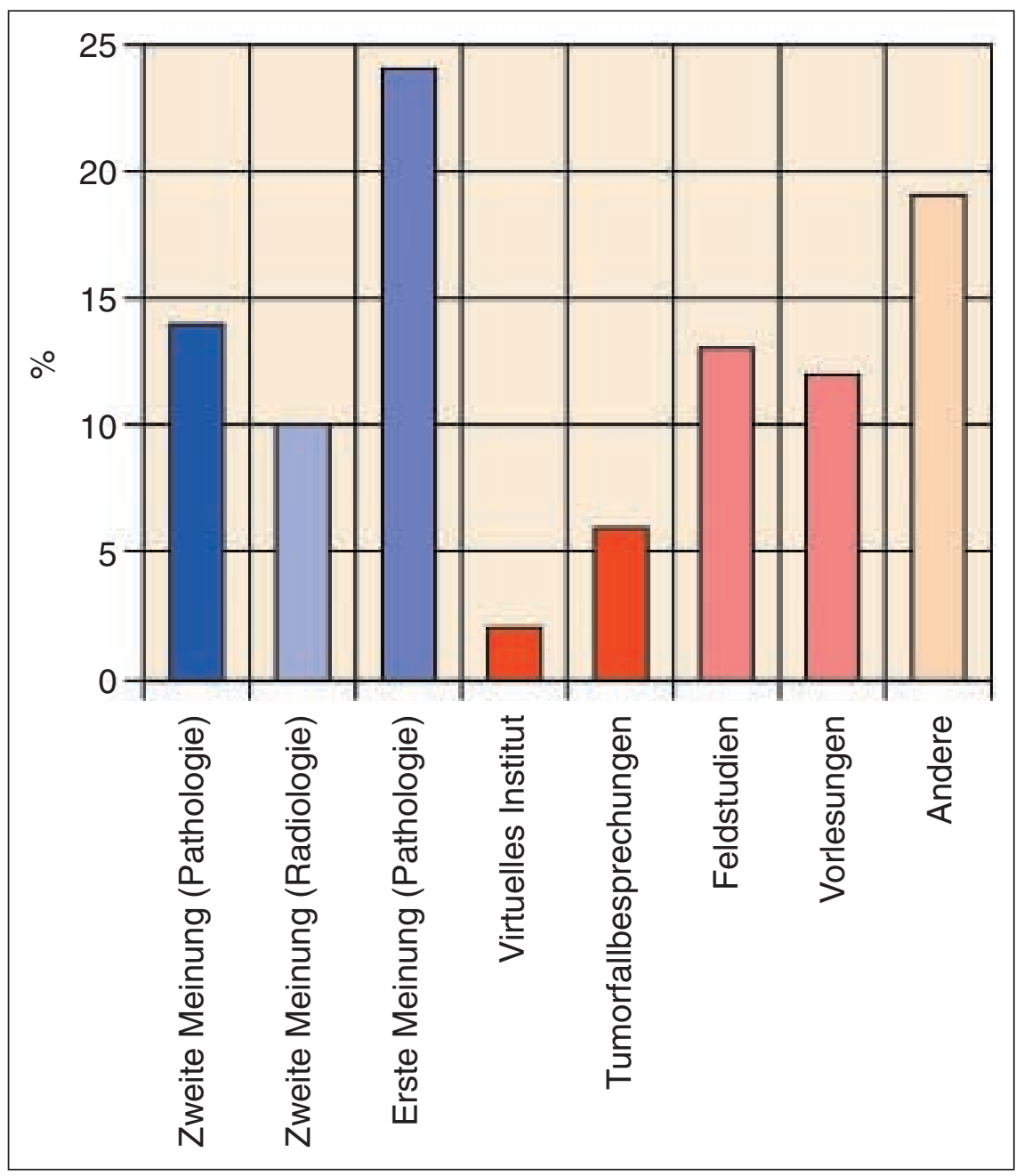

\section{Resultate}

Die verschiedenen Einsatzgebiete von iPath, welche sich in den ersten zwei Jahren ergeben haben, sind in Abbildung 3 zusammengestellt. Interessant ist die Beobachtung, dass iPath in $50 \%$ der Fälle nicht für pathologisch-anatomische Diagnosen verwendet worden ist, sondern für Tumorfallbesprechungen, Feldstudien, Vorlesungen und Diskussionen unter Spezialisten (in der Säule «Andere» enthalten). Die intraoperativen Schnellschnitte mit dem Kreisspital Samedan [3], welche ebenfalls mit iPath durchgeführt werden, sind nicht mitgezählt und werden deshalb auch nicht mitdiskutiert.
Für die pathologisch-anatomische Diagnostik zugunsten zweier Spitäler (in Honiara, Solomon Islands, und in Dhaka, Bangladesh) ist seit 6 Monaten ein virtuelles Institut in Betrieb. In diesem Institut leisten sechs Pathologinnen und Pathologen nach einem vereinbarten Wochenplan Dienst. Drei der Mitglieder dieses Instituts sind pensioniert und arbeiten demzufolge $\mathrm{zu}$ Hause. Pro Woche treffen in diesem Institut im Durchschnitt acht Fälle dieser beiden Spitäler zur Diagnostik ein. Dem virtuellen Institut werden $\mathrm{ab}$ und $\mathrm{zu}$ aber auch andere als histologische Befunde zur Diskussion zugesandt (z. B. endoskopische und dermatologische Befunde).

Seit über einem Jahr wird iPath in der Onkologiebesprechung des Kreiskrankenhauses Lörrach (Baden-Württemberg, Bundesrepublik Deutschland) eingesetzt. Die Schlüsselinformationen zur Klinik der Fälle und die Fragestellungen werden von den Onkologen im Feld «Description» (Abb. 2) eingetragen. Die relevanten radiologischen, zytologischen und histologischen Befunde werden von den Pathologen (in Basel oder Freiburg im Breisgau) und den Radiologen (am Kreiskrankenhaus oder in der Praxis) in der Bildgalerie («Images», Abb. 2) deponiert; das Beschlussprotokoll wird unter «Comments» (Abb. 2) festgehalten.

Der Einsatz von iPath in Feldstudien ist möglich geworden, seit ebenfalls Formulare als Dokumente in die Bildgalerie aufgenommen werden können. Diese Formulare können für alle Fälle einer bestimmten Gruppe exportiert werden. Als Resultat erscheinen alle Feldinhalte des einzelnen Formulars in einer zweidimensionalen Matrix, welche ihrerseits auf den lokalen Computer zur Weiterverarbeitung gespeichert werden kann. Mit den Formularen können - umgekehrt zur primären diagnostischen Intention der Telemedizin - Informationen von peripheren Institutionen an Zentren gebracht werden.

Seit einem Jahr steht eine «Kopie» von iPath als iTeach (http://teleteach.patho.unibas.ch) zur Verfügung und wird in der Lehre eingesetzt. iTeach stellt eine Plattform dar, auf welcher die Lehrenden ihre Lehrinhalte dynamisch, individuell und beliebig strukturiert den Lernenden zur Verfügung stellen können. Die Gruppen entsprechen in iTeach einzelnen Vorlesungen, Kursen oder Seminaren, die Fälle einzelnen Vorlesungskapiteln und die Gruppen von Objekten einzelnen Unterkapiteln (Abb.4). Die Lernenden können den Vorlesungsinhalt (Texte, Bilder, andere Unterlagen) zu jeder Zeit von jedem Ort aus einsehen. Wenn die Dozenten erlauben, dass auch Kolleginnen und Kollegen Dokumente zu 
Abbildung 4

Verwendung des Konzeptes von iPath in der Lehre.

iTeach ist das «Schwestersystem» von iPath für die Lehre. Weil die Lehrenden in der Lehre üblicherweise die Fragen stellen, spielen sie - aus theoretischer Sicht - die Rolle der Nichtexperten, und weil die Lernenden Antworten geben, diese die Rolle der Experten. Eine Gruppe entspricht einer Vorlesung, ein Fall einem Vorlesungskapitel (z.B. «Pathologie der Nebenniere») und Gruppen von Objekten einzelnen Unterkapiteln (z.B. «Nebennierenrinde»). Die Dialoge, welche mit der ChatFunktion geführt werden, werden automatisch zu den einzelnen Vorlesungskapiteln abgelegt.

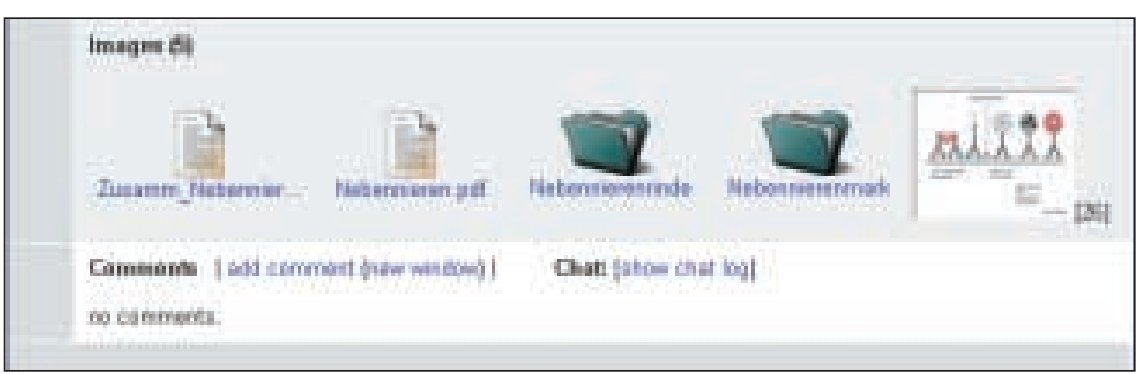

Abbildung 5

Benutzerfreundlichkeit moderner Telemedizinsysteme.

Moderne Telemedizinsysteme sollten übersichtliche Bildschirmseiten aufweisen, in welchen der Benützer sich einfach zurechtfinden kann. Die dem Benützer zur Verfügung stehenden Aktionsmöglichkeiten sollten klar ersichtlich sein.

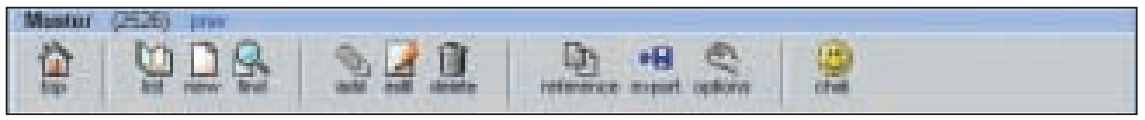

ihren Vorlesungskapiteln zufügen können, ist eine Ringvorlesung möglich. Die für eine Vorlesung registrierten Personen (Lehrende und Lernende) können sich gegenseitig mit der Funktion «Chat» über die Lehrinhalte austauschen oder Kommentare zu den Lehrinhalten der einzelnen Kapitel abgeben (unter «Comments»).

\section{Diskussion}

Internet hat die Telepathologie und die Telemedizin revolutioniert [2, 4-13]. Mit dieser neuen Technologie ist es viel einfacher geworden, Expertenwissen und Erfahrungen, welche in Zentren vorhanden sind, in Gegenden zu transferieren, wo dieses Wissen nicht verfügbar ist [14-20]. iPath entspricht einem solchen neuen «Verteilsystem». Es basiert auf dem Prinzip der freien Software und kann dementsprechend auf beliebig vielen Servern, welche unabhängig voneinander operieren, eingesetzt werden.

Von seiner Eigenschaft als offenes System her kann iPath möglicherweise dazu beitragen, den in letzter Zeit im Gesundheitswesen zu beobachtenden Paradigmenwechsel zu verstärken. Unter dem Begriff «Paradigma» [21] wird eine bestimmte Konstellation von Überzeugungen,
Werten und Verfahrensweisen verstanden. Der erwähnte Paradigmenwechsel in der Medizin dürfte vor allem die Kollaboration in der Medizin betreffen. Ein möglichst einfach zu bewerkstelligender und geregelter Austausch von Bildinformationen $[22,23]$ ist zu einer wichtigen Voraussetzung für eine effiziente Kollaboration geworden. Das Konzept von iPath mit seinem modularen Aufbau erfüllt diese Bedingung.

Bei der Entwicklung von iPath wurde nach dem kleinsten gemeinsamen Vielfachen gesucht, welches mit einem einzigen System möglichst viele verschiedene Applikationen zulässt. Es zeigte sich dabei, dass dieses kleinste gemeinsame Vielfache technisch aus folgenden Komponenten besteht: relationaler Datenbank, Betriebsmodulen, freier Software als Programmierungstool und Linux als Betriebssystem. Funktionell lässt sich die Telemedizin als Telekollaboration verstehen und auf einen strukturierten Dialog ohne Hardware- und Softwarerestriktionen zwischen einem Nichtexperten, welcher fragt, und verschiedenen Experten, welche antworten, reduzieren.

Der Begriff iPath steht nicht nur für das Instrument iPath, sondern auch für ein Konzept. Dieses Konzept folgt den Rahmenbedingungen eines «content management system». Das Potential von iPath als offenes System wurde von einigen Autoren [24] bereits unmittelbar nach dessen Publikation im Internet (http://telepath. patho.unibas.ch) erkannt. Weil iPath neben der passiven Telekonsultation [25] auch die aktive Telekonsultation [1, 26-29] erlaubt, wird es sowohl den «Hybridsystemen» [30] als auch den semidynamischen Systemen [24] zugerechnet. Von einer aktiven Telekonsultation wird gesprochen, wenn sich die beiden Gesprächspartner gleichzeitig und direkt (telefonisch oder über die in iPath verfügbare Chat-Funktion) über die Bildinhalte austauschen, andernfalls handelt es sich um eine passive Telekonsultation. Die Experten deponieren ihre Meinungen in beiden Situationen jeweils im Feld «Comments» des besprochenen Falles.

Die Ansprüche der Benutzer an telemedizinische Systeme sind hoch. Die Systeme werden daran gemessen, ob sie benutzerfreundlich $\mathrm{zu}$ bedienen sind (Abb. 5), ob sie sich funktionell einfach an neue Aufgabenstellungen anpassen und ob sie sich problemlos betriebstechnisch aufrüsten lassen. Das wichtigste Mass zur Beurteilung von Telemedizinsystemen ist deren Modularität geworden [1, 31]. Wells und Sowter [32] kritisieren vor allem, dass Anpassungen von kommerziell erhältlichen Systemen durch die Hersteller zu lange dauern. Die bisherigen Er- 
fahrungen mit iPath zeigten, dass eine Parametrisierung unterschiedlicher Geräte und somit Anpassungen des Systems an spezifische Umgebungsbedingungen einfach möglich sind.

Grundsätzlich sollten elektronisch verschickte Daten kryptifiziert werden [33]. In iPath ist eine automatische Kryptifizierung verfügbar; die Länge des Schlüssels beträgt 40 Bit. Als weitere Prophylaxe gegen potentielle Angriffe auf iPath oder Komponenten von iPath werden Einzelkonsultationen nach einer vorgegebenen Zeit (Minuten) automatisch abgebrochen. Die Eingabe von patientenbezogenen Daten in iPath ist untersagt.

Die Kosten für das Telepathologiesystem sind immer wieder Gegenstand von Diskussionen gewesen [7, 34-37]. Die einzigen Kosten, welche aktuell spezifisch anfallen, wenn iPath eingesetzt wird, sind die Kosten für die Bildquelle (mindestens eine Digitalkamera oder eine Videokamera mit Bildwandler), die Verbindung zwischen der Bildquelle (Kamera, Ultraschallgerät, Röntgenarchiv, andere Geräte) und einem Computer und die Kosten für den Datentransfer über das gewählte Netzwerk (Integrated Services Digital Network [ISDN], E-Mail, Internet). Es ist zu erwarten, dass in Zukunft eine einmalige Gebühr für das Einrichten einer Arbeitsgruppe auf einem Server (etwa Fr. 3000.-) und von jedem Benützer pro Jahr eine Gebühr für den Zugang zu iPath (etwa Fr. 700.-) verlangt werden wird.

In beiden Projekten (in der Schweiz mit dem Kreisspital Samedan und auf den Solomon Islands mit dem Referral Hospital Honiara) ging es um Forschungspartnerschaften in zwei ganz verschiedenen Umfeldern:

1. in einer informations-technologisch dicht versorgten Region und Gesellschaft und

2. in einem «Rural Country» mit minimaler Kommunikationsinfrastruktur. Empirisch liess sich klar nachweisen, dass eine als «open distributed system» und als «content management system» konzipierte Telemedizin in beiden Umfeldern das zu leisten vermag, was sich die Benützer von ihr versprechen.

Sie erlaubt es aber auch gleichzeitig, die beiden Umfelder sinnvoll und eng miteinander zu verknüpfen. Auf diese Weise kann Telemedizin die Gelegenheit bieten, für alle an der medizinischen Kollaboration Beteiligten einen echter Mehrwert zu generieren und eine Win-WinSituation zu schaffen: Den verschiedenen Nichtexperten werden ihre Fragen beantwortet; die Experten haben die Gelegenheit, ihren Erfahrungsschatz wertvoll $\mathrm{zu}$ erweitern. Weil die neuen Technologien jedem Nichtexperten er- lauben, die Meinung von Fachexperten ohne spezielle Vorinvestitionen auf der ihnen vertrauten Plattform einzuholen, könnte die Telemedizin zu einem Pfeiler einer sinnvollen Globalisierung der Medizin werden. Bei der Planung und Durchführung des Projektes «Honiara», welches als Synonym für «Telemedizin in Rural Countries» genommen werden kann, konnte beiläufig festgestellt werden, dass die elf von der Swiss Commission for Research Partnerships with Developing Countries (http://www.kfpe.ch) aufgestellten Postulate realistisch und erfüllbar sind.

\section{Literatur}

1 Oberholzer M, Fischer HR, Christen H, Gerber S, Bruhlmann M, Mihatsch M, et al. Telepathology with an integrated services digital network a new tool for image transfer in surgical pathology: a preliminary report. Hum Pathol 1993;24:1078-85.

2 Brauchli K, Christen H, Haroske G, Meyer W, Kunze KD, Oberholzer M. Telemicroscopy by the Internet revisited. J Pathol 2002;196:238-43.

3 Steffen B, Gianom D, Winkler C, Hosch HJ, Oberholzer M, Famos M. [Frozen section diagnosis using telepathology]. Swiss Surg 1997; 3:25-9.

4 Montironi R, Thompson D, Scarpelli M, Bartels HG, Hamilton PW, da Silva VD, et al. Transcontinental communication and quantitative digital histopathology via the Internet; with special reference to prostate neoplasia. J Clin Pathol 2002;55:452-60.

5 Brauchli K, Helfrich M, Christen H, Jundt G, Haroske G, Mihatsch M, et al. [The future of telepathology. An Internet "distributed system" with "open standards"]. Pathologe 2002; 23:198-206.

6 Szymas J, Wolf G, Papierz W, Jarosz B, Weinstein RS. Online Internet-based robotic telepathology in the diagnosis of neuro-oncology cases: a teleneuropathology feasibility study. Hum Pathol 2001;32:1304-8.

7 Leong FJ. Practical applications of Internet resources for cost-effective telepathology practice. Pathology 2001;33:498-503.

8 Gardziella S, Hufnagl P, Bodo M, Mortara I, Dietel M. [The UICC Telepathology Consultation Center. "Second opinion" over the Internet for pathologists]. Pathologe 2001;22:288-9.

9 Petersen I, Wolf G, Roth K, Schluns K. Telepathology by the Internet. J Pathol 2000; 191:8-14.

10 Okada DH, Binder SW, Felten CL, Strauss JS, Marchevsky AM. "Virtual microscopy" and the internet as telepathology consultation tools: diagnostic accuracy in evaluating melanocytic skin lesions. Am J Dermatopathol 1999; 21:525-31. 
11 Della Mea V. Telepathology and the Internet. Telemed Today 1999;7:17-18, 44.

12 Szymas J, Wolf G. Telepathology by the internet. Adv Clin Path 1998;2:133-5.

13 Wolf G, Petersen I, Dietel M. Microscope remote control with an Internet browser. Anal Quant Cytol Histol 1998;20:127-32.

14 Desai S, Ghosh TK, Chinoy R, Mohan A Dinshaw KA. Telepathology at tata memorial hospital, Mumbai and Barshi, a rural centre in Maharashtra. Natl Med J India 2002;15:363-4

15 Singh N, Akbar N, Sowter C, Lea KG, Wells CA. Telepathology in a routine clinical environment: implementation and accuracy of diagnosis by robotic microscopy in a one-stop breast clinic. J Pathol 2002;196:351-5.

$16 \mathrm{Gu}$ J. Recent advances in telepathology. Cell Vis 1998;5:62-6.

17 O'Brien MJ, Takahashi M, Brugal G, Christen H, Gahm T, Goodell RM, et al. Digital imagery/ telecytology. International Academy of Cytology Task Force summary. Diagnostic Cytology Towards the 21st Century: An International Expert Conference and Tutorial. Acta Cytol 1998;42:148-64

18 Martinez A, Villarroel V, Seoane J, Del Pozo F. EHAS Program: Rural telemedicine systems for primary healthcare in developing countries.

19 Wootton R. The possible use of telemedicine in developing countries. J Telemed Telecare $1997 ; 3: 23-6$

20 Wright D. Telemedicine and developing countries: A report of Study Group 2 of the ITU Development Sector. J Telemedicine Telecare $1997 ; 4: 1-85$

21 Küng H. Erkämpfte Freiheit. Erinnerungen. München; Pieper Verlag GmbH; 2002.

22 Belnap CP, Freeman JH, Hudson DA, Person DA. A versatile and economical method of image capture for telepathology. J Telemed Telecare 2002;8:117-20.

23 Loane M, Wootton R. A review of guidelines and standards for telemedicine. J Telemed Telecare 2002;8:63-71

24 Furness PN, Bamford WM. Telepathology. Review. Current Diagnostic Pathology 2001;7:281-91.
25 Weinberg DS, Allaert FA, Dusserre P, Drouot F, Retailliau B, Welch WR, et al. Telepathology diagnosis by means of digital still images: an international validation study. Hum Pathol 1996;27:111-8.

26 Nordrum II. Real-time diagnoses in telepathology. Adv Clin Path 1998:2:127-31.

27 Dunn BE, Almagro UA, Choi H, Sheth NK, Arnold JS, Recla DL, et al. Dynamic-robotic telepathology: Department of Veterans Affairs feasibility study. Hum Pathol 1997;28:8-12.

28 Nordrum I, Eide TJ. Remote frozen section service in Norway. Arch Anat Cytol Pathol 1995; 43:253-6.

29 Oberholzer M, Fischer HR, Christen H, Gerber S, Bruhlmann M, Mihatsch MJ, et al. Telepathology: frozen section diagnosis at a distance. Virchows Arch 1995;426:3-9.

30 Demichelis F, Barbareschi M, Boi S, Clemente C, Dalla Palma P, Eccher C, Forti S. Robotic telepathology for intraoperative remote diagnosis using a still-imaging-based system. Am J Clin Pathol 2001;116:744-52.

31 Szymas J. Teleeducation and telepathology for open and distance education. Anal Cell Pathol 2000;21:183-91.

32 Wells CA, Sowter C. Telepathology: a diagnostic tool for the millennium? J Pathol 2000;191:1-7.

33 Kuo RL, Aslan P, Dinlenc CZ, Lee BR, Screnci D, Babayan RK, et al. Secure transmission of urologic images and records over the Internet. J Endourol 1999;13:141-6.

34 Rogers N, Furness P, Rashbass J. Development of a low-cost telepathology network in the UK National Health Service. J Telemed Telecare 2001;7:121-3.

35 Bryant J. Cost minimization analysis of telepathology: a critical review. Am J Clin Pathol 2000;113:902-5

36 Agha Z, Weinstein RS, Dunn BE. Cost minimization analysis of telepathology. Am J Clin Pathol 1999;112:470-8

37 Tsuchihashi Y, Mazaki T, Murata S, Nakasato K, Morishima M, Nagata $\mathrm{H}$, et al. Telepathology and cytology in Kyoto, Japan, to support regional medicine, with special references to their need, accuracy and cost. Adv Clin Path 1998;2:131-2. 